\title{
APL JANUS System Progress on Commercial Suborbital Launch Vehicles: Moving the Laboratory Environment to Near Space
}

H. Todd Smith ${ }^{1}$, Ryan T. Hacala ${ }^{1}$, Erik M. Hohlfeld ${ }^{1}$, Weston K. Edens ${ }^{1}$, Charles A. Hibbitts ${ }^{1}$, Larry J. Paxton ${ }^{1}$, Steven P. Arnold ${ }^{1}$, Joseph H. Westlake ${ }^{1}$, Abigail M. Rymer ${ }^{1}$, Al Chacos ${ }^{1}$, Mason A. Peck ${ }^{1}$, Ben R. Zeiger ${ }^{2}$

'The Johns Hopkins University Applied Physics Laboratory, Laurel, MD; '2 Luxel Corporation, Friday Harbor, WA

Abstract

Multiple private companies are building suborbital reusable launch vehicles possessing vastly different designs. Many of these companies originally focused on space tourism; however, revolutionary applications for scientific and engineering research as well as technology demonstrations and instrument development are emerging. The dramatic reduction in cost over traditional launch systems as well as a guaranteed (and rapid) safe payload return enable many new launch vehicle applications. These new capabilities will essentially move the laboratory environment up to the edge of space. To make use of these novel launch vehicles, the John Hopkins University Applied Physics Laboratory has established a Commercial Suborbital Program with a core system (JANUS) to support and enable many future suborbital missions. This program has already conducted six suborbital flight missions to establish vehicle interfaces and analyze the suitability and limits of each flight environment. Additionally, this program has also been selected by the NASA Flight Opportunities Program for five additional operational suborbital missions. Here we present the results of our completed missions as well as descriptions of future selected missions scheduled for 2021-2023.

Keywords

Commercial Suborbital • JANUS • NASA Flight Opportunities • Blue Origin • Virgin Galactic • Masten Space Systems

\section{INTRODUCTION}

In recent years, several companies have actively developed both manned and unmanned fully reusable suborbital vehicles of various designs and capabilities to provide routine access to near-Earth space. As an indicator of their rise in prominence, there has been an upsurge in commercial spaceport infrastructure to support these vehicles. This has led to many states vying to establish spaceports to potentially host many of the planned suborbital activities. There has also been tremendous international interest, with spaceports being discussed or planned for overseas operations. This is clearly an exciting time to be involved in the growing suborbital industry. The exact nature of the flight path, the altitudes to be reached, and the duration of the flight vary for each suborbital Reusable Launch Vehicle (sRLV) vendor. In general, these vehicles achieve altitudes $>100 \mathrm{~km}$, and they provide on the order of a few minutes in a low gravity environment. Initially, sRLV companies solely targeted space tourism as their primary source of income. However, it has become evident that these new vehicles offer game-changing opportunities for scientific research, engineering development, and education as well.
First, the cost of flying payloads on these launch vehicles is over an order of magnitude lower than the traditional launch systems (Jurist, 2009). Such a reduction in cost facilitates space access for many smaller or nontraditional organizations as well as enables lower cost and more frequent opportunities. Next, sRLV companies are planning for routine and rapid flight cadence allowing for more responsive flight tests. Perhaps most significantly, these vehicles offer both a gentle launch and a safe return of payload, often only a few hours after the flight. This is an entirely new capability that enables flight of low cost, prototype payloads that can be flown iteratively, generally beyond what is achievable with NASA's traditional suborbital portfolio. Over the last 10 years, we have found that this capability is effectively raising the laboratory environment to the edge of space. This emerging capability also offers the opportunity to fly "humantended" payloads on these vehicles where researchers will be able fly with their experiments further creating an analogy to the laboratory environment as well as opening up new research strategies.

Many applications for these new spacecraft are only starting to evolve and emerge. The opportunities for revolutionary scientific

\footnotetext{
${ }^{\dagger}$ Corresponding author: $\mathrm{H}$. Todd Smith

E-mail: h.todd.smith@jhuapl.edu
} 
research at $\sim 100 \mathrm{~km}$ are truly novel. This atmospheric region, which reaches up to the lower edge of Earth's ionosphere, informally referred to as the "ignorosphere," has been difficult to explore using in situ instrumentation because it is too high for high-altitude balloons and aircraft, and too low for traditional orbiting spacecraft. Until now, only comparatively high cost and infrequent sounding rocket experiments have provided scientists with in situ measurements needed to quantify the composition and dynamics of the tenuous critical transition region between Earth's atmosphere and space-often without the option for functional payload recovery. Routine in situ research of this region will greatly increase our understanding of Earth's atmosphere and offer the possibility of opening up a research frontier on our figurative front doorstep.

These new spacecraft also offer avenues to significantly reduce the cost and development schedule of spacecraft instruments and technology demonstrations. Currently, spaceflight tests of novel instruments are relatively expensive and time-consuming because of the pedantic flight cadence and must be developed to survive harsher launch- and environmental conditions than in its final form as a satellite mission. With the existing portfolio of sounding rockets, the launch conditions are frequently the biggest obstacle to instrument development. The lower launch acceleration of these SRLVs and the soft return of payloads will allow scientists and engineers to fly early stage prototypes that can be modified based on flight test results and flown on a nearly arbitrary cadence, compared to the $\sim 2$ years cadence common to the sounding rocket program (Christe et al., 2016). This creates a low cost, iterative launch environment that is more like a laboratory than a traditional spaceflight. Additionally, even though these launch vehicles do not access the full range of space environments, almost all space instruments and payloads should increase their technology readiness level (TRL) from these flights, as they involve autonomous operation of the payload during a spaceflight test. In fact, these new spacecraft may help usher in a new assessment of flight readiness based on system level (Sauser et al., 2006). We have also found great operational and technical benefits from flying payloads even if the suborbital conditions are not completely relevant space environment due to the flight duration and altitude. Given the relatively large launch mass and volume capabilities of the new SRLVs, one could even envision a suborbital flight test of long-duration satellite payloads in final form prior to the actual space launch. Since the payload would be safely returned to the researcher, this opportunity could provide a final operational test and the opportunity to make improvements prior to the actual mission.

Above are just a few of the possible applications of these revolutionary spacecraft that are now starting to evolve. Members of the Johns Hopkins University Applied Physics Laboratory (JHU APL) have been actively planning for and participating in this surge of suborbital activities over the past 10 years. Through these activities, APL has developed closer relationships with vendors that help educate these companies on the significant research and engineering potential of their launch vehicles as well as helping design vehicle interfaces to more efficiently and effectively accommodate these activities. This led APL to establish a Commercial Suborbital Program (CSP) that is designed to accommodate novel, rapid, and low-cost activities (Arnold and Smith, 2013). The resulting program is enabling the APL to derive maximum benefit by rapidly exploiting SRLVs for scientific research, engineering development, and technology demonstration.

\section{MOTIVATION AND STRATEGY OF THE JHU APL COMMERCIAL SUBORBITAL PROGRAM}

The APL CSP is designed to minimize the obstacles for the application of the novel benefits afforded by SRLVs by using a common architecture for power, control, and data logging. Traditional relatively high spaceflight payload costs and long development timelines-much of which is devoted to tasks controlled by JANUS - tend to negate the low cost and rapid cadence advantages of commercial reusable launch vehicles, and these barriers are particularly high for new entrants in the field who might have, for example, optical expertise but not payload systems engineering expertise. Therefore, the goal of this program is to maximize the low cost, iterative, and rapid potential of SRLVs by removing these barriers. We wish to treat spaceflight more as laboratory activities rather than typical more costly and time-consuming deployment. To enable this goal, the APL CSP is establishing a regular launch schedule for technology and instrument development, research, and regular testing of internal research and development projects, which also includes serendipitous opportunities to fly internal research experiments at little or no cost.

The core element of the APL CSP is the JANUS platform (named after the ancient Roman god of gateways and new beginnings), which provides core reusable payload capabilities. This universal core platform is a small physical enclosure that provides common payload and experiment components and capabilities. This base payload is designed to reduce the complexity, timeline, and cost of conducting research, instrument development, and technology demonstration on emerging commercial spacecraft. JANUS provides power, data storage, instrument control, ambient environment characterization, and the electrical and physical interface to the spacecraft for instruments and experiments. Its low cost, iterative design is completely self-contained providing multiple power and data interfaces. This reduces cost, complexity, and schedule because separate and complete payloads are not required for each commercial mission. In keeping with the 
low-cost strategy, the JANUS platform is being developed in an iterative fashion with limited funding. As we fly missions, we improve JANUS capabilities as needed and defined through flight testing.

The current JANUS version (2.1) is a modular and stackable integration sub-payload that allows for expansion to support multiple experiments on Masten Space Systems, Virgin Galactic, and Blue Origin sRLVs. For commercial suborbital missions where the unit is returned, this payload can be completely self-contained, consisting of a flight battery, harnesses, low-voltage power supply (LVPS) electronic board, and a processor board. The dimensions of JANUS 2.1 are $19.6 \times 5.7 \times 15.2 \mathrm{~cm}$ with a mass of $1.4 \mathrm{~kg}$ (Figure 1 ). The current JANUS 2.1 design includes: $\pm 15 \mathrm{~V},+5 \mathrm{~V},+3.3 \mathrm{~V}$ and high-current $+5 \mathrm{~V}$ power interfaces; $128 \mathrm{~GB}$ flash data storage; a high rate USB 2.0 interface for ground testing; and many additional instrument interfaces (up to 24 customizable $<= \pm 10 \mathrm{~V}$ analog channels, up to $32 \mathrm{Rx}$ or $24 \mathrm{Rx} / 8 \mathrm{Tx} 3.3 \mathrm{~V}$ and $45 \mathrm{~V}$ GPIO, up to $8 \mathrm{Rx}$ or $4 \mathrm{Rx} / 4 \mathrm{Tx} 3.3 \mathrm{~V}$ and $25 \mathrm{~V}$ Rx only universal asynchronous receiver/transmitter (UART) format, and up to $5 \mathrm{Rx} / 3 \mathrm{Tx}$ RS-232 channels). Many of these channels can be modified at little to no cost to RS-422 or lowvoltage differential signaling (LVDS) formats.

To adequately control payloads and fully understand experiment results, JANUS is designed to characterize and monitor the local environment. Therefore, JANUS 2.1 contains 3D accelerometers and an inertial measurement unit $(3 \mathrm{D} \pm 8 \mathrm{~g}$ at $6.66 \mathrm{kHz}$ or $3 \mathrm{D}$ accelerometer and Inertial Measurement Unit (IMU) at $1.66 \mathrm{kHz}$ ) to observe launch, flight, and landing conditions as well as characterize the microgravity environment. Magnetic field observations provide not only important insight into the Earth's magnetic field but also characterization of potential electromagnetic interference (EMI) from other payloads or the spacecraft. To meet this requirement, JANUS 2.1 also includes a Vector Vaquier-type fluxgate magnetometer measuring a dynamic range of \pm 0.6 Gauss (0.001 uV/Gauss sensitivity) with at sampling rate of up to $3.5 \mathrm{kHz}$. Additionally, the JANUS 2.1 platform contains onboard pressure and temperature sensors. The LVPS board (Figure 1A) controls and modulates power to the experiments as well as the JANUS processor board and is contained within the JANUS chassis. It contains an EMI filter with slow turn-on control to minimize the power transients experienced by the SRLV. It also contains two housekeeping analog-to-digital converter (ADC)s that are used to monitor the current and voltage of each DC-DC module and can individually enable and disable each supply channel as needed for power reduction. The processor board has two opto-isolators to monitor external power sources and is also contained within the JANUS 2.1 chassis (Figure $1 A)$ enclosure. It contains a reprogrammable Xilinx Spartan 6 field-programmable gate array (FPGA) with a LEON
Processor, $200 \mathrm{MHz}$ crystal oscillator, and a power-on reset circuit. This board contains $128 \mathrm{~GB}$ of flash memory, a UART, a FTDI USB controller, and a JTAG/SPI for test purposes, and it can store up to three software versions in $2 \mathrm{MB}$ of onboard MRAM that can be selected at start up. All of the previously mentioned instrument and spacecraft data interfaces can be found on this board as well.

The current JANUS platform is an example of its own merits of the rapid iteration enabled by a fast flight cadence: it is the third version of the JANUS platform, with its design iteratively improved through flight tests on SRLVs. This platform supports a three-phase strategy for the APL CSP. We are currently completing Phase 1 with the development of the JANUS platform, establishing interfaces for multiple spacecraft, and assessing the applicability, limitations, and suitability of the flight environment. So far, this phase has involved six successful flight missions (on Blue Origin, Virgin Galactic, and Masten Space Systems vehicles-Figure 1B) funded internally and by the NASA Space Technologies Mission Directorate (STMD) Flight Opportunities Program (FOP). After two more flight tests (tentatively scheduled for the Blue Origin NS-13 and Virgin Galactic 1st Qtr. FY 2021 flights), we anticipate initiating the next phase of operations. Phase 2 involves transition to operational suborbital flight experiments and development activities using JANUS to support and enable these payloads. The NASA FOP has currently selected and funded seven more APL sRLV missions to conduct experiments or technology demonstrations as well as establish advanced vehicle interfaces to enable access to the external spacecraft environment. Once the APL CSP Phase 2 is established, we will also initiate Phase 3 intended to expand into suborbital support of larger scale flight testing and NASA missions as well as nontraditional sponsors.

\section{JHU APL COMPLETED FLIGHT EXPERIMENTS}

JANUS 2.1 has been designed, developed, and tested by the support from the NASA FOP as well as APL internal funding. As of the time of writing, the JANUS system has successfully flown on six suborbital flights with an additional five missions selected by NASA for flight over the next 3 years. Below we provide summaries of these completed missions.

\section{1st Mission: Risk-Reduction Flight for 1st FOP-Funded Payload (Masten Space Systems sRLV)}

The initial JANUS 1.0 system (Figure 2) was first selected for a suborbital test flight through the NASA FOP in 2011. This design was intentionally modest and inexpensive, intended to validate new capabilities enabled by commercial suborbital spacecraft and gain experience with these spacecraft. Our plan was to fly an early prototype that, with guaranteed 

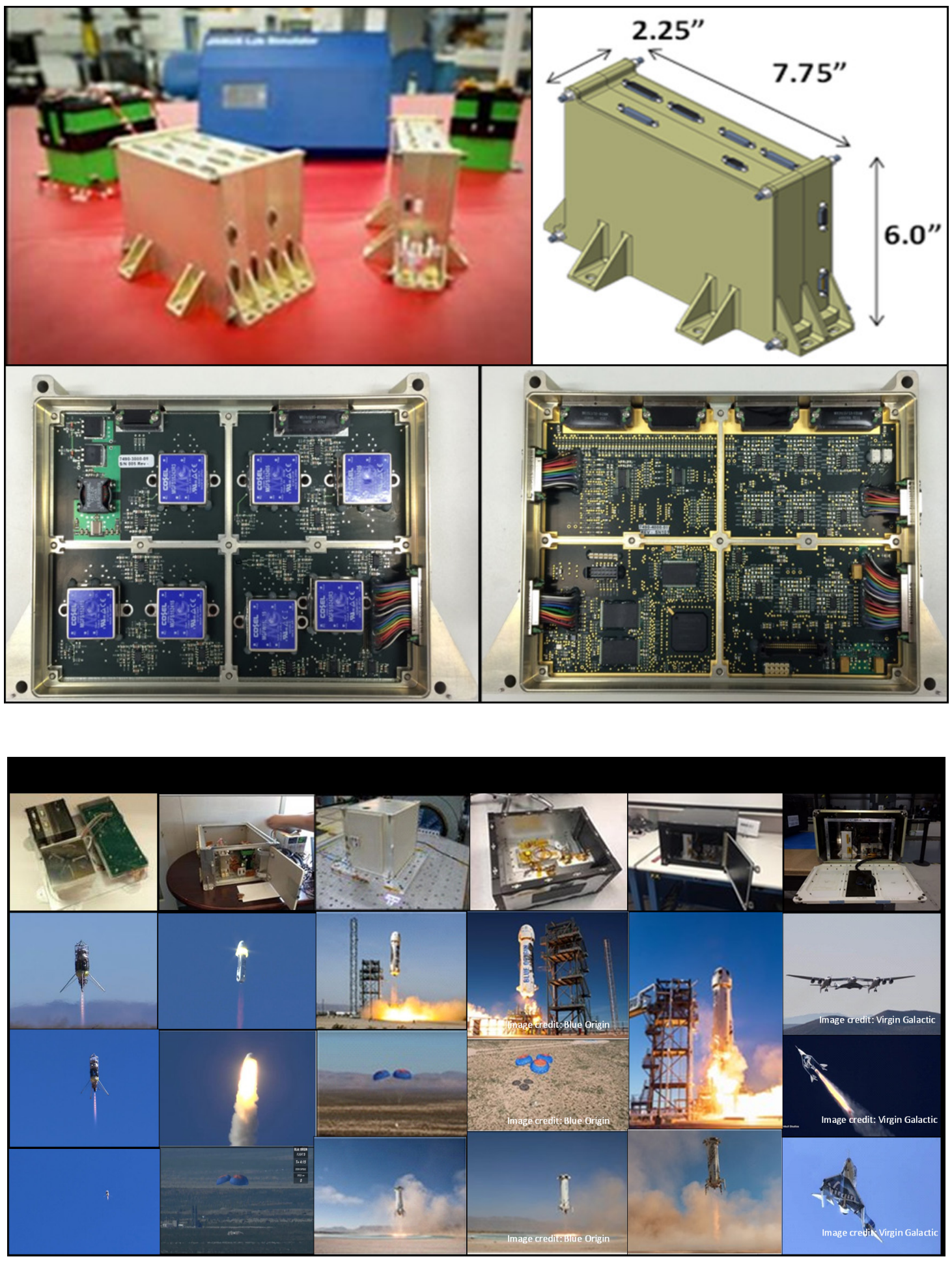

Figure 1. (Top panel) JANUS 2.1 system. Top left: Double (left) and single (right) units with associated flight batteries and ground support system (middle). Top right: JANUS 2.1 dimensions. Bottom left: Low-voltage power supply electronics board. Bottom right: processor electronics board. (Bottom panel). Images from the six completed JANUS mission (by column from left to right). Top row shows the JANUS payload for each mission with flight images below each of these columns. 


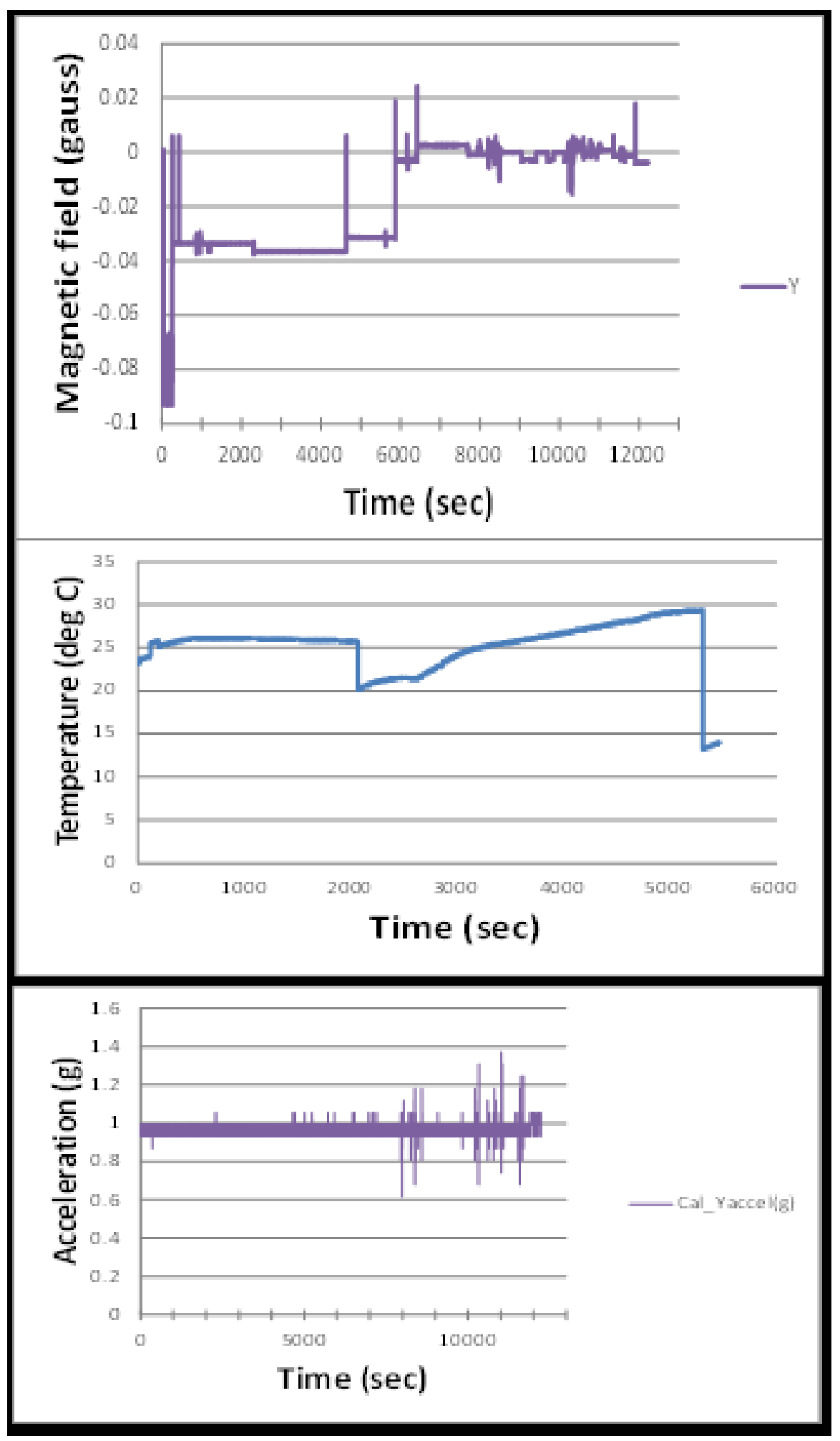

Figure 2. 1st mission on the Masten Space Systems Xombie sRLV. Top: Magnetic field observations. Middle: Thermal observations of the JANUS processor board. Bottom: Total acceleration observations. 
safe return of the payload, would be improved based on inflight performance. Thus, we applied our intended iterative approach to dramatically reduce the development cost of JANUS by supplementing ground testing with exposure to a more relevant flight environment. APL collaborated with NASA to conduct a low-altitude "risk-reduction" flight while awaiting higher-altitude operational flights that provide an earlier opportunity to further test and improve this design. Although the actual launch of our 2nd mission (discussed later) happened slightly before this flight (resulting from early stage launch date uncertainties), this risk-reduction mission process was initiated earlier and considered our first mission. Additionally, our 2nd mission included design improvements based on the early stages of this mission.

For this risk-reduction campaign, Masten Space Systems performed three activities with our payload at the Mojave Air and Spaceport in California on the Xodiac vertical takeoff vertical landing (VTVL) sRLV (Figure 2). The $3.7 \times 1.8 \mathrm{~m}$ unmanned vehicle is powered by a regeneratively cooled liquid oxygen/isopropyl alcohol rocket engine and primarily used to test Lunar and Martian landing technologies. However, this sRLV can also perform low-altitude payload test flights. On October 24, 2016, our payload was integrated on the Xodiac launch vehicle and powered up for a static ground power test. Then on October 27, 2016, our payload was integrated and powered up for a tethered flight test. Finally, on November 2, 2016, our payload was integrated and powered up for the operational low-altitude free-flight test, which attained an altitude of $\sim 0.5 \mathrm{~km}$ above ground level (AGL; Figure 2). Although these were very limited flights, the tests provided a low-cost method of initial JANUS system flight testing that allowed to dramatically improve the magnetometer design.

This test flight validated the pressure and thermal detections systems, and identified the critical need for accelerometer and magnetometer upgrades. Figure 2 shows the magnetic field, total acceleration, and thermal data collected during the free-flight test. This risk-reduction flight was very successful as it indicated that the accelerometers and magnetometers were not sufficient to meet mission requirements. It is possible that several of these needs for improvement would have been missed with ground qualification, because isolated test environments only test for known conditions rather than comprehensively including all flight conditions. This flight was also successful in testing autonomous, remote flight operations, in which it identified several critical issues that were corrected. Based on these results, we dramatically improved the payload design of JANUS 2.0, helping improve the chances of success during an operational mission. In addition to making the payload smaller (the new JANUS version was reduced to $21 \%$ of the volume and $28 \%$ of the mass of JANUS 1.0) and more robust, we integrated a much better magnetometer as well as adding vector accelerometers, a vector IMU, and an improved thermal sensor. We upgraded the power supply to a Blue Origin systems with significant flight heritage. Thus, even this modest flight activity noticeably improved the JANUS design and validated the application of SRLVs in a low cost, rapid, and iterative development program.

\section{2nd Mission: Risk-Reduction Flight for 1st APL-Funded Payload (Blue Origin sRLV Escape Test)}

Concurrently with the above NASA FOP flight activities, APL also conducted a self-funded flight test to assess JANUS acceleration-based trigger capabilities on the Blue Origin New Shepard sRLV. This SRLV is also a VTVL rocket designed to carry up to six passengers to altitudes over $100 \mathrm{~km}$. It is approximately $18 \mathrm{~m}$ tall and composed of a pressurized crew capsule (CC) with six large windows that integrates on top of a propulsion module (PM). The entire vehicle launches using a single BE-3 liquid hydrogen/liquid oxygen (LH2/LOX) cryogenic engine producing $\sim 100,000 \mathrm{lbf}$ of thrust. The main engine cuts off approximately $2.5 \mathrm{~min}$ after launch, and then the CC mechanically separates from the PM. Both components coast up to their respective apogees under momentum. After a few minutes of freefall, the PM autonomously returns to the landing pad approximately $1.5 \mathrm{~km}$ from the launch pad. The CC also performs a soft landing under parachutes and retrorockets to a landing zone nominally within $8 \mathrm{~km}$ of the launch pad. The first New Shepard test flight successfully returned the CC; however, the PM crashed. To date, two New Shepard SRLVs have flown a combined 11 fully successful flights.

Unfortunately, the launch date for our operational flight test slipped by over a year from October 2016 to 2017 because our scheduled flight was reassigned as an "escape test" to assess the vehicle capabilities to protect human life during a flight anomaly. Not only would this flight not follow a nominal flight profile, but there was an expectation that the propulsion module may be damaged or destroyed during this test. This flight was designated "M6" by Blue Origin (referring to it as the 6th launch of the New Shepard). We took advantage of this situation to conduct a risk-reduction test of our own on this flight while awaiting our nominal operational flight in 2017. For this flight (and all subsequent Blue Origin missions so far), our payload was mounted inside of a $40.6 \times 33.0 \times 25.3 \mathrm{~cm}$ enclosed payload locker integrated into one of six payload "stack" locations inside of the pressurized CC. Each stack is capable of accommodating up to six single payload lockers.

On October 5, 2016 (Figure 3), JANUS flew successfully on the Blue Origin New Shepard launch vehicle from the West Texas Launch Site. Although this flight was not designed to reach maximum suborbital altitude, it involved a flight test of the escape motors that subjected our payload to much greater launch and vibrational loads than nominal conditions 


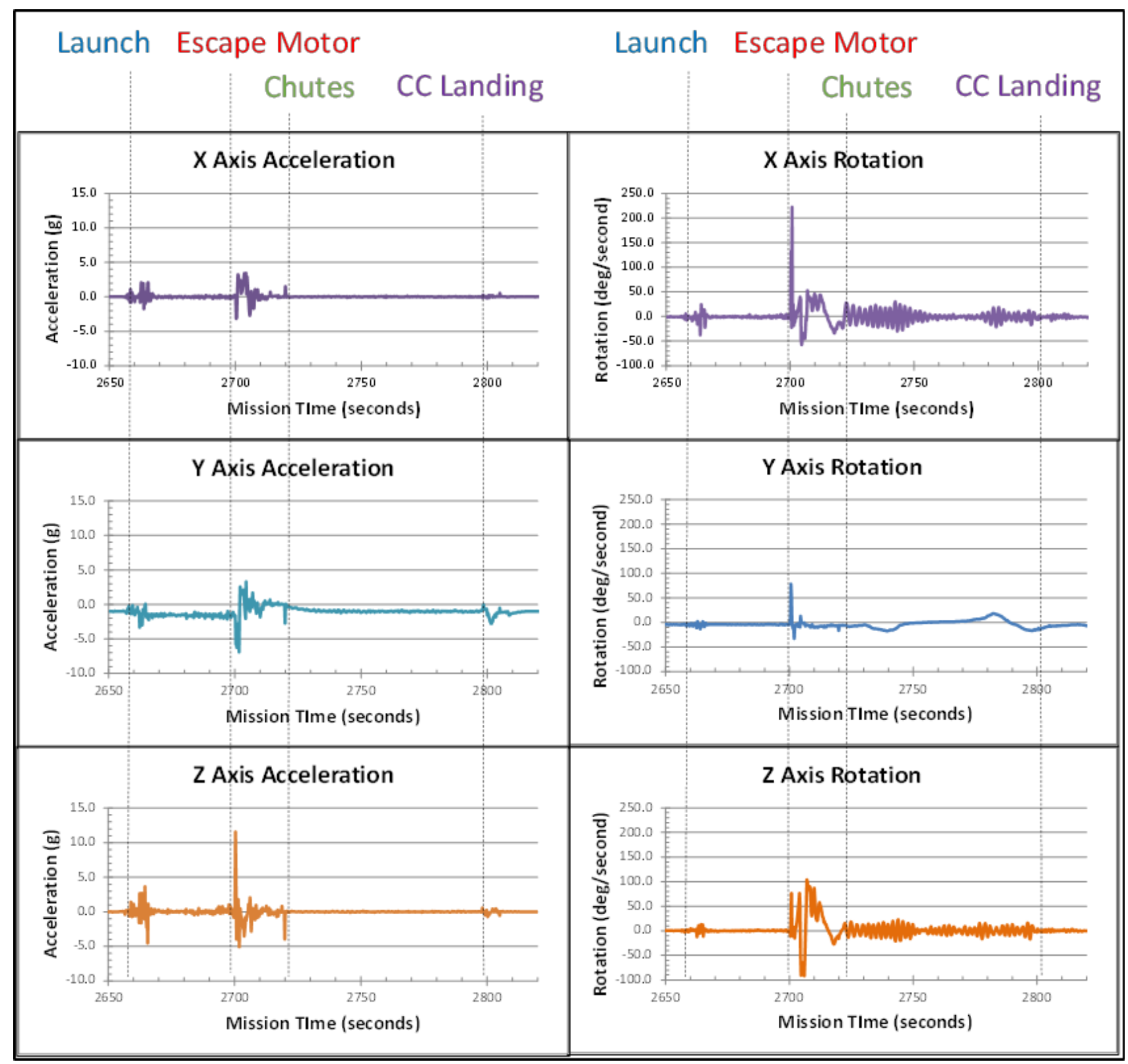

Figure 3. 2nd sRLV mission on the Blue Origin New Shepard CC escape motor test. Left column: Acceleration $(g)$ observations $(y$-axis vertical in CC, $x$ and $z$ axes orthogonal to $y$-axis). Right column: Rotational (deg/s) observations ( $y$-axis vertical in CC, $x$ and $z$ axes orthogonal to $y$-axis).

(by greater than a factor of 3 ) and reduced our ground testing requirements. Approximately $45 \mathrm{~s}$ after launch, the escape motors of the New Shepard CC successfully separated it from the PM and safely returned to the landing area. Interestingly, the PM also survived the escape test, continued to a nominal apogee and safely returned to the landing pad. This risk-reduction flight for JANUS comprehensively replaced our standard suite of expensive and time-consuming environmental testing required for the next launch, thus demonstrating the value of low cost, iterative development enabled by sRLVs.

The JANUS payload performed successfully, without any anomalies and exceeded all testing requirements under much more extreme flight conditions than those anticipated during operational flight. Figure 3 also shows the acceleration and rotation observations collected by JANUS during this flight, which were accurately captured across all phases of the flight. These data show that even under the most extreme flight 


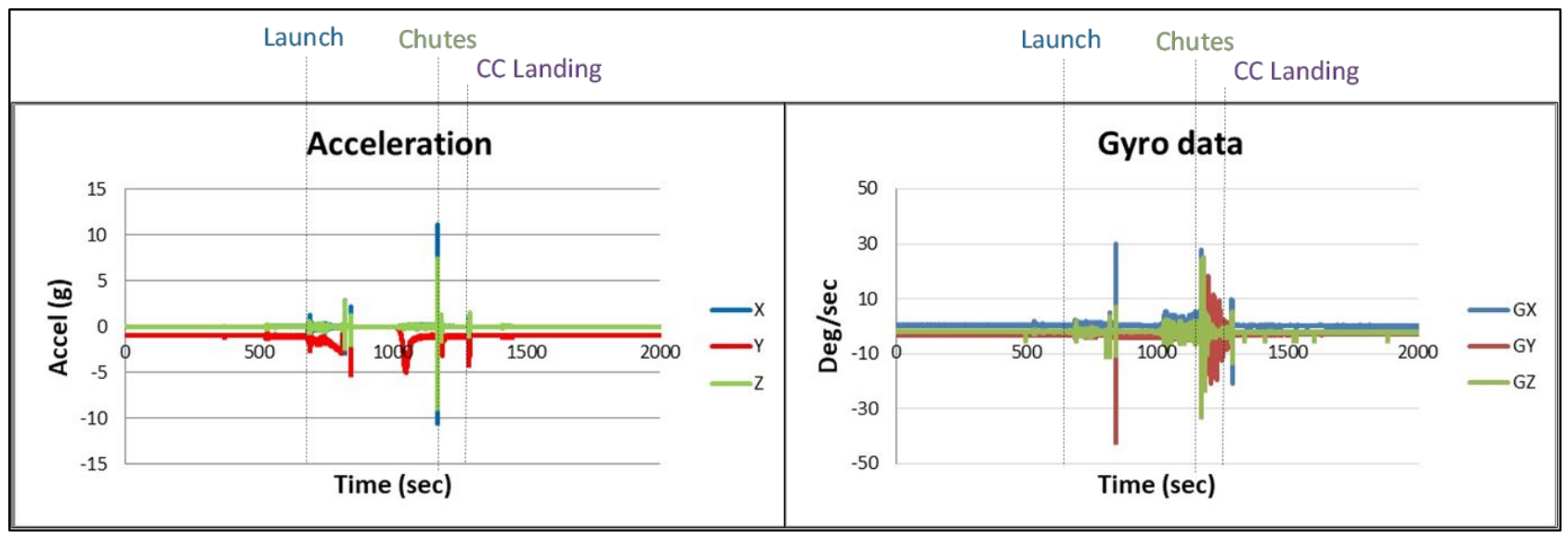

Figure 4. 3rd sRLV mission on the Blue Origin New Shepard sRLV. Left: Acceleration $(g)$ observations $(y$-axis vertical in CC, $x$ and $z$ axes orthogonal to $y$ ). Right: Rotational (deg/s) observations ( $y$-axis vertical in CC, $x$ and $z$ axes orthogonal to $y$-axis).

conditions, this vehicle presents a relatively gentle launch environment with a very soft landing. This flight activity also allowed the APL CSP to greatly improve its flight operations procedures, dramatically reducing the risk for future operational flights. Thus, these results help demonstrate our assertion that SRLVs can be essentially utilized as a low-cost laboratory in near space once regular and frequent flight cadences are established.

\section{3rd Mission: First Operational Flight Test of JANUS (Blue Origin SRLV)}

The next JANUS suborbital flight test was the operational APL-funded flight related to the previous risk-reduction flight, and it was the first to attain nominal altitude for the Blue Origin New Shepard. This flight offered APL its first opportunity to analyze nominal flight conditions of the New Shepard vehicle to assess its applicability for future research and engineering flights.

Blue Origin's New Shepard "M7" mission was launched on December 12, 2017, from the West Texas Launch Site (Figure 4). In addition to improving flight heritage for the upgraded JANUS 2.0 design and characterizing the ambient payload environment, we also conducted 35 accelerationbased experiments to test JANUS's ability to identify all changes in the flight environment correctly. The $\mathrm{CC}$ reached an altitude of $99.3 \mathrm{~km} \mathrm{AGL}$ during this flight with a total flight time of $603 \mathrm{~s}$. The capsule landed less than $1.5 \mathrm{~km}$ from the launch pad, with a maximum descent velocity of Mach 2.94. The PM, after reaching a maximum descent velocity (Mach 3.74), successfully reignited its engines and landed safely on the landing pad. JANUS 2.0 (Figure 4) performed successfully throughout the entire flight and continued to operate in low-power mode after landing. The payload was recovered successfully within two hours of landing; data analysis at APL showed all JANUS sensors and functions operated successfully, with all 35 acceleration-based triggers flawlessly tracking all phases of flight. Figure 4 shows the acceleration and rotation data collected during this flight with key flight events annotated. These data were collected at a high time resolution of $4 \mathrm{kHz}$, once again demonstrating the benign flight environment and very soft $\mathrm{CC}$ landing. Based on this flight, we were able to verify flight readiness for our first NASA FOP-funded operational flight and make minor design improvements to JANUS 2.1.

\section{4th Mission: First Magnetic Field Experiment (Blue Origin sRLV High-Altitude Escape Test)}

Following the above launch activities, APL was able to conduct its first NASA STMD FOP- funded operational mission. The flight objective of the proposed experiment was to characterize the ambient electromagnetic field environment in the CC to assess external and internally generated fields. Additionally, we improved the payload design of JANUS 2.1 by integrating the above fluxgate magnetometer and upgrading vehicle physical and electrical interfaces. This flight test ("P6" referring to the 6th flight containing payloads) occurred during the July 18, 2018, "high-altitude escape test" of the Blue Origin New Shepard launch vehicle at the West Texas Launch Site (Figure 5). This high-altitude escape test involved ignition of the CC escape motors after separation from the Propulsion Module. This activity resulted in the CC attaining a much higher than nominal altitude of $118.8 \mathrm{~km} \mathrm{AGL}$, thus allowing us to make observations at altitudes higher than those attained during normal flight.

JANUS 2.1 performed very well and we successfully measured the Earth's magnetic field from inside of the $\mathrm{CC}$ 


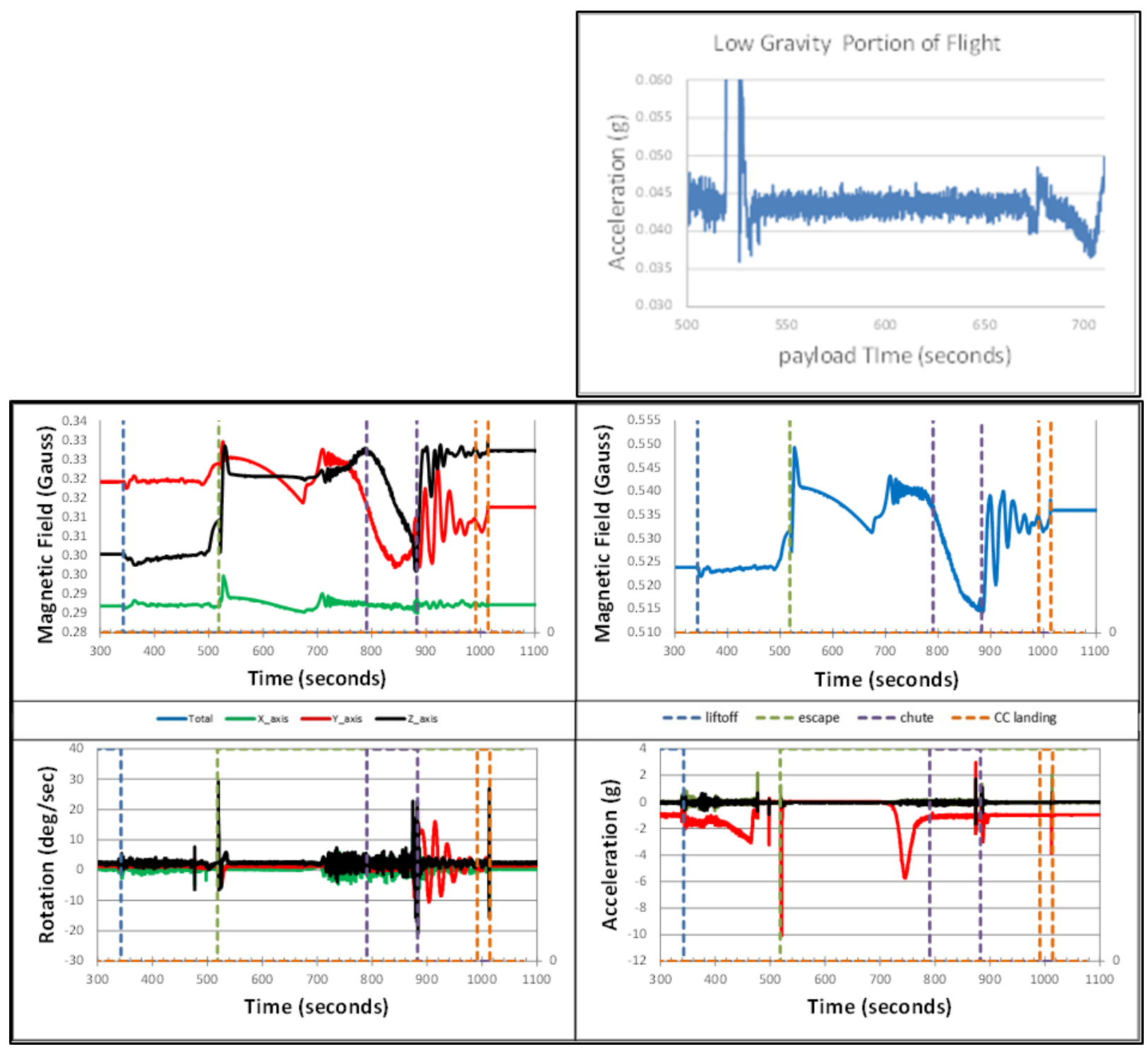

Figure 5. 4th mission on the Blue Origin New Shepard SRLV. Left middle: Magnetic field observations by axis. $y$-axis vertical in CC, $x$ and $z$ axes orthogonal to $y$-axis. Bottom middle: Rotation (deg/s) observations. Top right: Micro-gravity period analysis $(g)$. Right middle: Total magnetic field observations (gauss). Bottom right: Acceleration $(g)$ observations by axis.

(Figure 5). The magnetometer data show the total and axial magnetic fields observed during the flight test with key events (launch, escape motor firing, parachute deployment, and capsule landing) annotated on each figure. Prior to launch, we observed the unperturbed Earth's magnetic field from inside the CC (and inside a payload locker). Our initial observations are very stable and at an expected value $(0.524 \pm 0.001$ gauss). These observations are valuable because they show that it is possible to observe the Earth's magnetic field with great accuracy from inside of the $\mathrm{CC}$. During the flight, overall magnetic field observations are consistent with expectations with the exception of relatively minor perturbations (on the order of $<0.01$ gauss) during the escape test portion of the flight, likely caused by undetermined EMI produced during this test. Other customer payloads on this flight could also contribute to this observed EMI but were not individually recorded. This EMI appears to dissipate after the parachutes deploy. Finally, we observed some oscillatory EMI across two axes while the orientation of the $\mathrm{CC}$ changes during final descent. After landing, the magnetic field stabilizes once again.

This flight test also validated the improved acceleration and gyro observational capability of JANUS 2.1, allowing us 


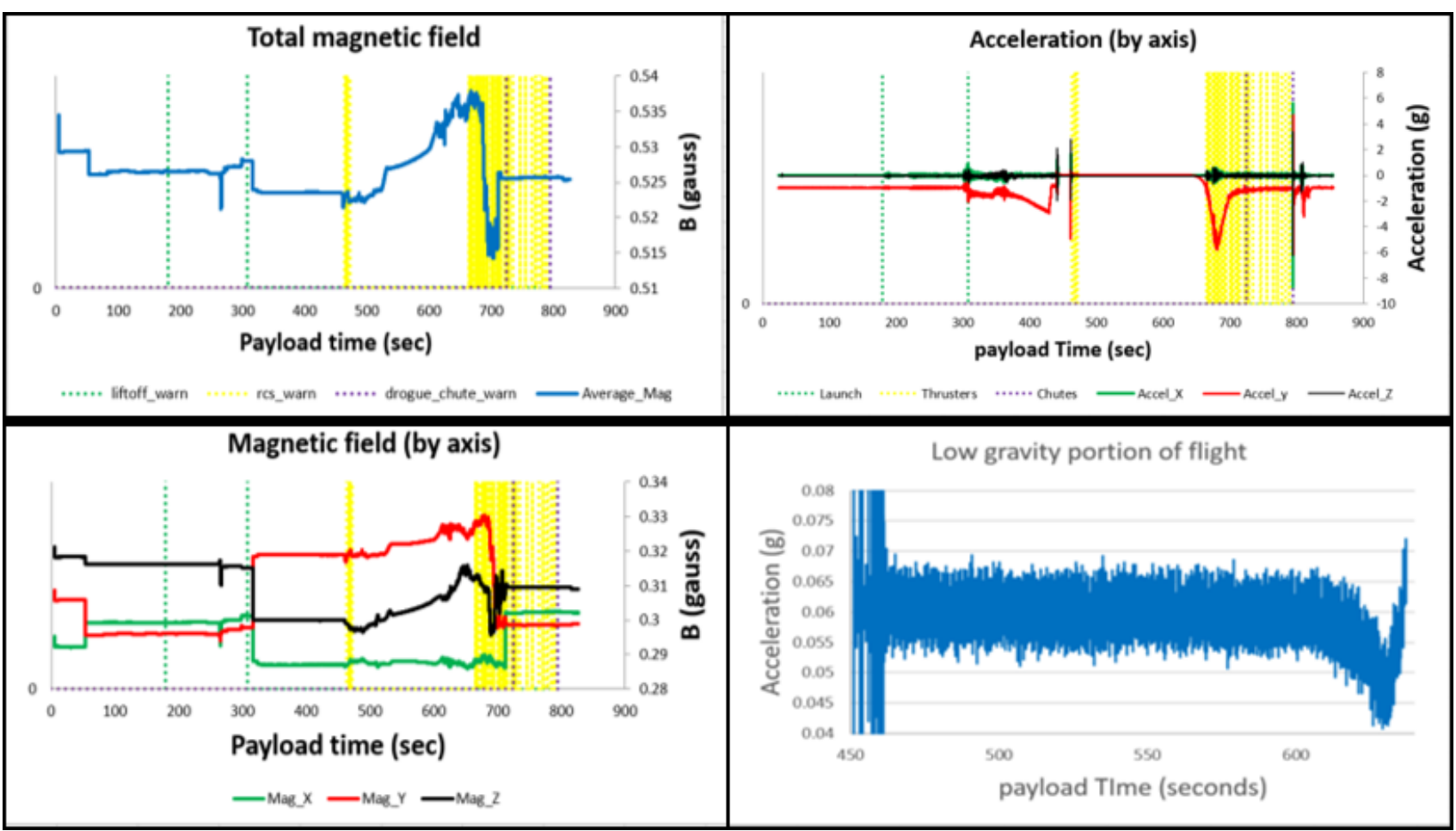

Figure 6. 5th sRLV mission on the Blue Origin New Shepard vehicle. Top left: Total magnetic field (gauss) observations. Bottom left: Axial magnetic field (gauss) observations. Top right: Axial acceleration $(g)$ observations. Bottom right: Low-gravity field $(g)$ observations. $y$-axis vertical in CC, $x$ and $z$ axes orthogonal to $y$.

to assess the quality of the micro-gravity portion of the flight. Figure 5 also shows the measured microgravity environment. Once the acceleration stabilized at or below $0.05 \mathrm{~g}$, we record an average $0.043 \pm 0.002 \mathrm{~g}$ for a duration of $182.03 \mathrm{~s}$ (this variation is likely a combination of thruster firings and measurement noise). JANUS 2.1 performed successfully during this flight and exceeded all test requirements during a higher than nominal flight profile resulting from the additional escape motor acceleration.

\section{5th Mission: Second Magnetic Field Experiment (Blue Origin sRLVN Flight)}

Following the previous flight test, APL conducted its first operational, nominal flight profile test of the JANUS system (funded by NASA FOP). The goal of this flight and the JANUS 2.1 configuration were similar to the previous flight; however, this flight followed the nominal profile expected during future flights. This flight test occurred on a Blue Origin New Shepard P7 launch out of the West Texas Launch Facility on January 23,2019 . The flight proceeded successfully, reaching an altitude of $107 \mathrm{~km}$ with safe landing of the CC and PM (Figure 6). This was our first opportunity to examine magnetic fields and microgravity inside of the New Shepard CC during a nominal flight profile.
JANUS 2.1 characterized the flight environment with a successful measurement of the Earth's magnetic field from inside of the CC, and we were able to observe perturbations to this field as a result of specific spacecraft activities and maneuvers (Figure 6). However, some small perturbations were unexpectedly different from our P6 observations. While some differences can be explained by the escape motor activities on P6, the sources of other differences have not been fully identified but are likely the result of other payload or vehicle instrument activity. This may indicate that for experiments sensitive to EMI, a pre-flight test activity with all payloads operating with magnetometer observations may be beneficial. Another remediation step may be to populate certain flights with only low EMI payloads.

Figure 6 shows our magnetometer data with total and axial magnetic fields observed during the flight test annotated with key events (launch, reaction control thruster events, and parachute deployment) in the figure. Prior to launch, we observed the unperturbed Earth's magnetic field from inside the CC (and inside a payload locker), which was stable $(0.526 \pm 0.002$ gauss). These figures show that after launch, the magnetic field slightly decreases as expected from the increase in altitude. Figure 6 can be compared with the magnetic field profile we observed during P6 (Figure 5). 
The basic profiles are similar, but there are some unexpected differences. As expected, the CC experienced noticeable immediate EMI during the P6 escape motor test and then dropped off (from $\sim 500 \mathrm{~s}$ until $\sim 700 \mathrm{~s}$ ), verified by the lack of this feature in the P7 data. Interestingly, during both flights, the total observed magnetic field steadily increases around apogee, then dramatically drops off, and recovers to what appears to be the ambient Earth's magnetic field. Even more curious is that this feature appears to occur after chute deployment during P6 but before chute deployment (after apogee) on P7. Although we have not identified the source, we hypothesize this event is EMI-related. Additionally, during P6, after chute deployment, there are relatively large magnetic field oscillations that do not appear during P7. This feature is likely caused by EMI from other payloads as it seems unlikely that that the high-altitude escape test flight would cause EMI during this phase. These results illustrate that electromagnetic interference occurs during different flight phases and must be accounted for when either measuring the Earth's magnetic field or conducting experiments that are sensitive to EMI. We intend to continue analyzing these data with respect to axial orientation, including in the context of and combined with future flight data, to more accurately determine the sources of EMI during flight. Additionally, in the future we will mount JANUS externally on the launch vehicle, and we can then compare observations to determine the absolute impact of the CC on magnetic field observations as well as the ability to measure external magnetic fields. This work can eventually lead to an assessment of spacecraft charging and the measures required to make accurate external observations.

Figure 6 also shows our acceleration observations for the payload $x-, y$-, and $z$-axis with key events (launch, RCS firings, parachute deployment) annotated on the figure. The data show the load experienced during launch as well as reentry, which is very similar to the P6 flight. Additionally, we were able to again assess the microgravity environment. Once the acceleration is stabilized at or below $0.07 \mathrm{~g}$, JANUS recorded an average $0.059 \pm 0.005 \mathrm{~g}$ for $185.99 \mathrm{~s}$. Interestingly, the previous escape test flight (P6) achieved slightly lower and more stable gravity than this nominal flight. This is likely because the CC attained a higher altitude into a slightly more rarified region during the high-altitude escape test.

\section{6th Mission: First Virgin Galactic sRLV Magnetic Field Experiment}

Our next JANUS flight test was similar to the previous one with the exception that it was flown on the Virgin Galactic SpaceShipTwo (VSS Unity), which is designed to carry two pilots and six passengers up to altitudes of $\sim 110 \mathrm{~km}$. This sRLV design is radically different from the Blue Origin New Shepard, and it will likely be suitable for different applications.
The vehicle is a suborbital spaceplane that is air launched from the dual fuselage White Knight Two aircraft at an altitude of approximately 50,000 feet. Once released, SpaceShipTwo is powered by a single hybrid rocket engine up to apogee. It then applies a feathered wing reentry system and lands on a standard runway. Virgin Galactic was in an earlier stage of flight testing than Blue Origin and so this flight was not intended to reach nominal altitudes. However, this provided our first opportunity to flight test JANUS on SpaceShipTwo to analyze flight conditions for this completely different type of sRLV.

This flight test occurred at the Mojave Air and Space Port in Mojave, California, on February 22, 2019 (Figure 7). VSS Unity performed successfully reaching an altitude of $89.9 \mathrm{~km}$ AGL and a top speed of Mach 3.0. In addition to carrying JANUS (as well as other payloads), this was the first flight with a human test passenger. JANUS 2.1 payload performed very well and we successfully recorded environmental data during all aspects of the test flight.

Figure 7 shows our vector acceleration observations as well as the total magnetic field compared to vehicle altitude, with data recorded at a $1.67 \mathrm{kHz}$ cadence. Such a high rate measures short impulses and may give the appearance of higher accelerations than would actually be perceived by flight participants as each observed value only represents a $6^{\prime} 10^{-4} \mathrm{~s}$ time period. Additionally, payload integration on this early stage flight is still under development, so APL was required to develop a custom payload container that integrated into the current VSS Unity payload locker using custom struts (Figure 7). These struts were an expedient way of integrating our payload but may have impacted the acceleration profile on the payload. We intend to examine this design and develop a more robust integration system for future flights if needed. However, these data accurately represent the current payload environmental conditions and constitute JANUS's first observations of a SpaceShipTwo flight profile. After White Knight 2 takeoff (at $\sim 1800 \mathrm{~s}$ ), our payload experienced a low-level acceleration until the peak acceleration after the SpaceShipTwo rocket ignition (at $\sim 4500 \mathrm{~s}$ ), followed by the microgravity period. Once the acceleration stabilized at or below $0.1 \mathrm{~g}$, we recorded an average $0.057 \pm 0.015 \mathrm{~g}$ for $162.03 \mathrm{~s}$ (Figure 7). We anticipate that this environment may become more stable as the spacecraft achieves more nominal, higher altitudes. After this time period, acceleration increased dramatically during initial descent and then reduced until it spiked again during landing. We also observed minor accelerations during taxi back to the hanger. Figure 7 also shows total acceleration compared to velocity profiles. Additionally, JANUS's Inertial Measurement Unit showed minor oscillatory rotations, likely introduced by our custom strut interface to the payload locker. 


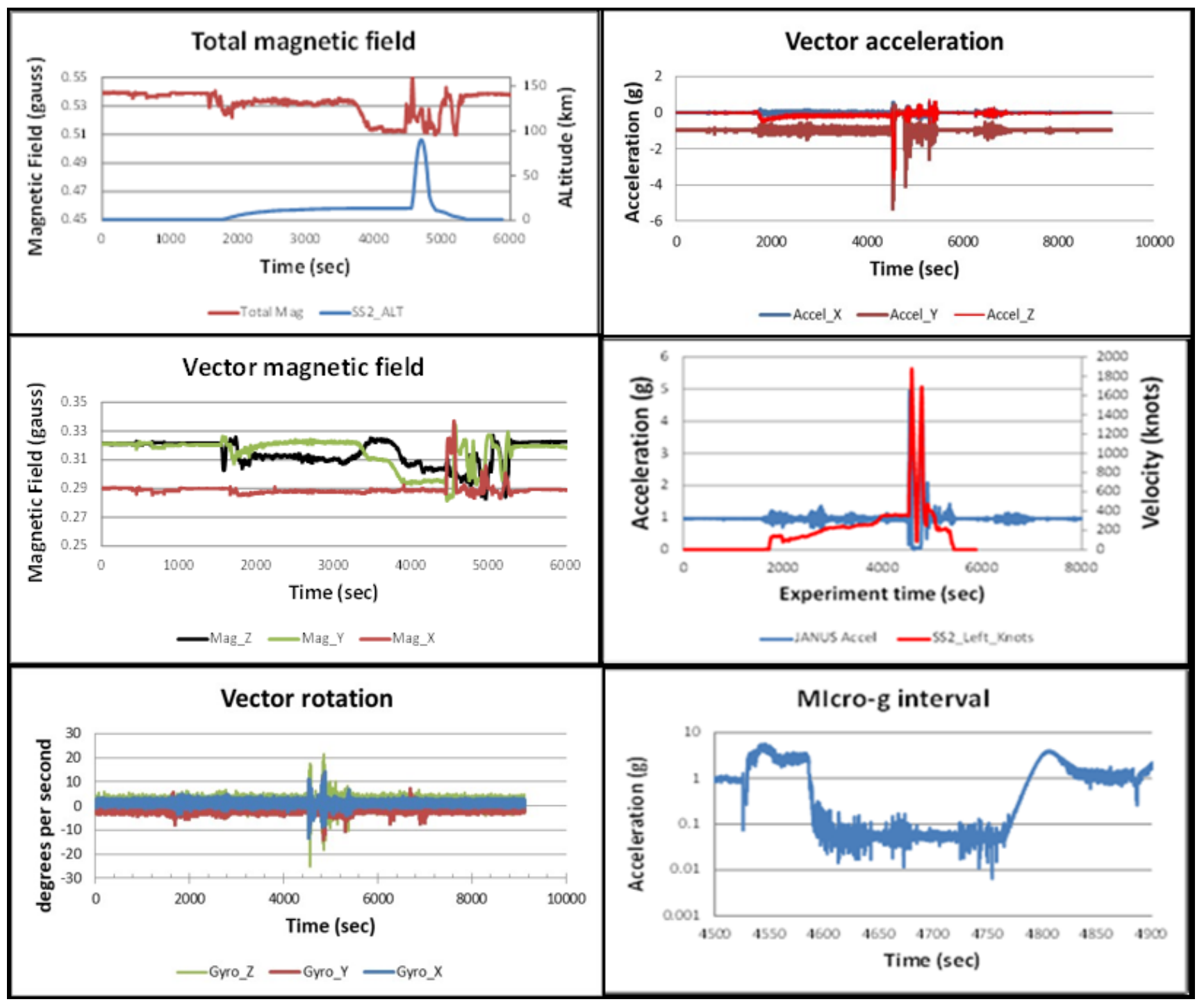

Figure 7. 6th sRLV mission on the Virgin Galactic SpaceShipTwo. Top left: Total magnetic field (gauss) observations compared to vehicle altitude $(\mathrm{km})$. Middle left: Vector magnetic field (gauss) observations. Bottom left: Vector rotation (deg/s) observations. Top right: Vector acceleration $(g)$ observations. Middle right: Total acceleration $(g)$ compared to vehicle velocity (knots). Bottom right: Low-gravity field $(g)$ observations. $y$-axis vertical in $\mathrm{CC}, x$ and $z$ axes orthogonal to $y$ axis.

Figure 7 shows magnetometer observations from the flight, as well. These data show the total and axial magnetic fields observed during the flight test relative to altitude and acceleration. Prior to takeoff, we adequately observed the Earth's magnetic field from inside the spacecraft (and inside a payload locker) and was very stable at an expected value, measured at $0.5391 \pm 0.0005$ gauss. This reading was the same before and after JANUS was placed inside the spacecraft, thus indicating that vehicle itself is not significantly interfering with the observations. However, there is noticeable EMI during takeoff as well as during powered ascent, the microgravity phase, and initial descent. We need to work further with Virgin Galactic to attempt to isolate the sources of EMI. Of note, the payload $x$-axis magnetic field is very stable with several sharp peaks after separation, likely indicating spacecraft EMI (this direction is in the vertical plane of the vehicle). It is possible that some observed EMI is actually from other payloads rather the spacecraft itself. Therefore, as with our Blue Origin flights, we will gain much more insight by comparing these results with future flights. 


\section{JHU/APL SCHEDULED FLIGHT EXPERIMENTS}

NASA FOP has selected and funded multiple future APL sRLV missions that will transition our program from flight environment assessment and suitability (Phase 1) to technology demonstrations and scientific research (Phase 2). The APL CSP is currently scheduled to fly JANUS on the next Virgin Galactic SpaceShipTwo flight to analyze the acceleration and magnetic field environments for a nominal flight profile, which should provide insight into the suitability of this SRLV for various applications. JANUS is also manifested to fly on the next Blue Origin New Shepard launch to establish a direct connection between our GPS receiver and the spacecraft GPS antenna. The new capability will combine with our IMU to provide for much more accurate inflight position and velocity information as well as the possible measurement of atmospheric Total Electron Content (TEC) on all future flights (Sukkarieh et al., 1999; Dyrud et al., 2007; Copper et al., 2019) and crosses over into the CSP Phase 2. Such observations could provide unique results as these data have never been available at suborbital altitudes at the frequency and resolution enabled by multiple JANUS flights. With successful completion of these flight tests, the JANUS platform will be ready to support operational research and engineering missions on both Blue Origin and Virgin Galactic sRLVs. Additionally, the above successfully completed missions have validated the ease at which the JANUS system can inexpensively and rapidly support multiple experiments. Below, we briefly describe the NASA FOP-selected SRLV missions that JANUS is scheduled to fly from 2021 to 2023 .

\section{Establishing Access to the External Spacecraft Environment}

In these previous missions, the JANUS 2.1 has flown inside of the pressurized New Shepard CC or SpaceShipTwo fuselage. However, many scientific payloads require direct access to space. Thus, the first mission of the APL CSP Phase 2 involves establishing payload access to the external spacecraft environment. In situ measurements at the altitude range 60 $120 \mathrm{~km}$ are so sparse because it is too high for conventional aircraft and balloons yet too low for orbiting satellites. To date, only sparse and infrequent rounding rocket observations exist for this region. For example, Barucci et al. (2011) outline the great need to characterize the billions of meteoroids that enter Earth's atmosphere each day (Ceplecha et al., 1998) and assess their impact on our atmosphere. External environment access provides the opportunity to directly sample meteoricbased aerosols greater than $10,000 \mathrm{~cm}^{-3}$ near $100 \mathrm{~km}$ (Hunten et al., 1980; Cziczo et al., 2001).

Another scientific application involves studying the complex and variable ion chemistry in Earth's upper atmosphere. Despite charged particles in this region being critical for maintaining our atmosphere and causing significant impact such as high-altitude lighting, we have made limited progress in quantifying their variability, either spatially or temporally. Remarkably, we appear to have more measurements of negative ions in the ionosphere of Saturn's moon Titan from the NASA Cassini mission than we do of Earth's ionosphere (Rymer et al., 2009; Smith and Rymer, 2014).

There are also numerous examples of benefits for NASA and commercial end users for technology development enabled by SRLV missions. In these cases, new technologies or instrument designs can be rapidly developed or advanced to market with flight heritage through external access on low cost, frequent launches. The below follow-on missions help illustrate some such examples. There are many more infusion possibilities for end users enabled by external access than these examples below, but these help to illustrate the far reaching applications, with many more certainly emerging as external environment access capability is established.

However, establishing external access capability is very challenging as it is difficult to integrate a payload outside of the vehicle without increasing risk related to the capsule/ fuselage structure and aerodynamics, and these sRLVs are designed primarily as commercial human transports rather than conveyors of scientific payloads. Fortunately, after considerable collaboration with Blue Origin, we were able to identify a location on top of the PM that could accommodate an external payload which is extremely well suited for both Blue Origin and APL (Figure 8). First, the risk to the $\mathrm{CC}$ is minimized as the payload will be placed on the PM. Additionally, this location places external payloads in vehicle ram facing direction during ascent, which is ideal for sampling the external environment and collecting particles. This is also a good location for observing upward. Since the maximum PM altitude is close to that attained by the $\mathrm{CC}$, little performance is lost by this payload relocation. Additionally, during initial descent, this payload location will be in the anti-ram orientation, which may be better suited for payload deployments (as our ChipSat mission discussed later).

Our next scheduled JANUS mission will involve flying on top of the New Shepard PM to establish an interface for external payloads and to analyze the flight environment from this location. After successful completion of this mission (tentatively scheduled for 3rd quarter of 2021), APL will begin the operational phase (Phase 2) of its CSP involving research, technology demonstration, and instrument testing and validation.

\section{Technology Demonstration of Vertically Aligned Carbon Nanotubes for Radiometers}

The APL CSP will conduct its first technology demonstration flight test once the above external environment access interface becomes operational. The goal of this test is to 


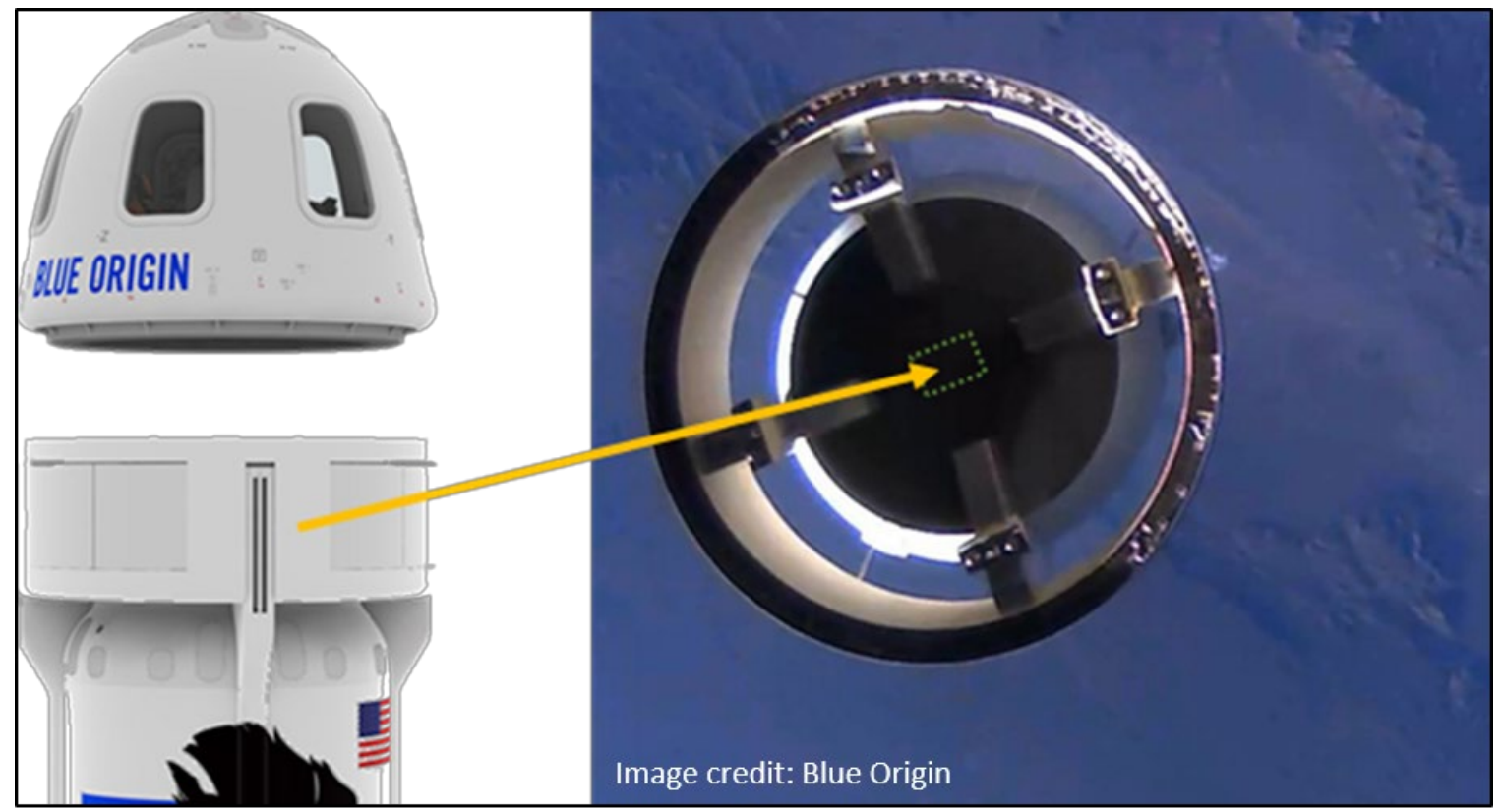

Figure 8. Payload location on top of the Blue Origin New Shepard PM for external space environment access.

demonstrate a vertically aligned carbon nanotubes (VACNT)based radiometer technology on a commercial SRLV. The results of this flight demonstration would raise the TRL, validating their proposed use in future NASA and operational Earth science missions.

We intend to use sRLVs as a low cost, rapid platform for improving VACNT radiometers based on the lessons learned from the RAVEN mission. VACNT-based bolometer radiometers can provide instrument improvements needed to make Earth- and Sun-observing radiometers sufficiently accurate and low cost to measure Earth's absolute energy balance/imbalance for the first time. This is precisely the measurement that is needed to resolve the climate change debate and enable vastly superior predictions of future change. Thus, our suborbital testing mission will provide a low-cost opportunity to prepare such detectors for future operational missions.

VACNTs are very promising material development in space technology over the last decade (Figure 9). They are cylindrical carbon structures aligned perpendicular to a substrate, and they are considered the blackest known substance to humankind (Yang et al., 2008; Mizunoa et al., 2009). Additionally, VACNTs do not outgas or cause contamination, are mechanically robust, and have an extremely large thermal conductivity (Sample et al., 2004; Xu and Fisher, 2006; Cola et al., 2007). Because of their unique electrical characteristics, they have been suggested for future use in numerous space applications as radiometric absorbers, stray light suppressors, emitters for thermal management, sources of electrical generation and transmission, and superlight structures. External environment testing and validation on SRLVs would provide a cost-effective, rapid method of verifying flight survivability and thermal response in a near space relevant environment.

We are motivated to pursue this particular application of VACNTs because two advancements are needed to measure the absolute radiation imbalance thought to drive climate change: a minimum of $20^{\circ}-30^{\circ}$ wide field of view radiometers encircling the globe and affordable-highly accurate radiometers. Our VACNT radiometer design (Figure 9) is based on the one recently flown on the NASA ETSO RAVEN CubeSat technology demonstration mission (Swartz et al., 2019). The sensor weighs 145 grams with a diameter of 2 inches and a height of 2.125 inches. It is constructed primarily of Aluminum 6061 and Copper OPHC with a sapphire window. The VACNTs were grown on a $1 \mathrm{~cm}^{2}$ wafer affixed to a $4 \% \mathrm{Ag}$ substrate. This sensor uses $+5 \mathrm{~V}, 15 \mathrm{uF}$, and $80 \mathrm{~mA}$ (analog) and +3.3 V, $15 \mathrm{uF}$, and max $35 \mathrm{~mA}$ (digital) connections, which are easily provided by JANUS. The field of view is 127 steradians. The radiometers are designed to cover the flux range from direct solar measurements $\left(1361 \mathrm{~W} \mathrm{~m}^{-2}\right)$ down to those encountered viewing the Earth $\left(100-750 \mathrm{~W} \mathrm{~m}^{-2}\right)$ with a precision of $<0.01 \mathrm{~W} \mathrm{~m}^{-2}$. The receiver and heatsink temperatures are shown in Figure 9 as a function of time to be compared to observations recorded during the flight test. 


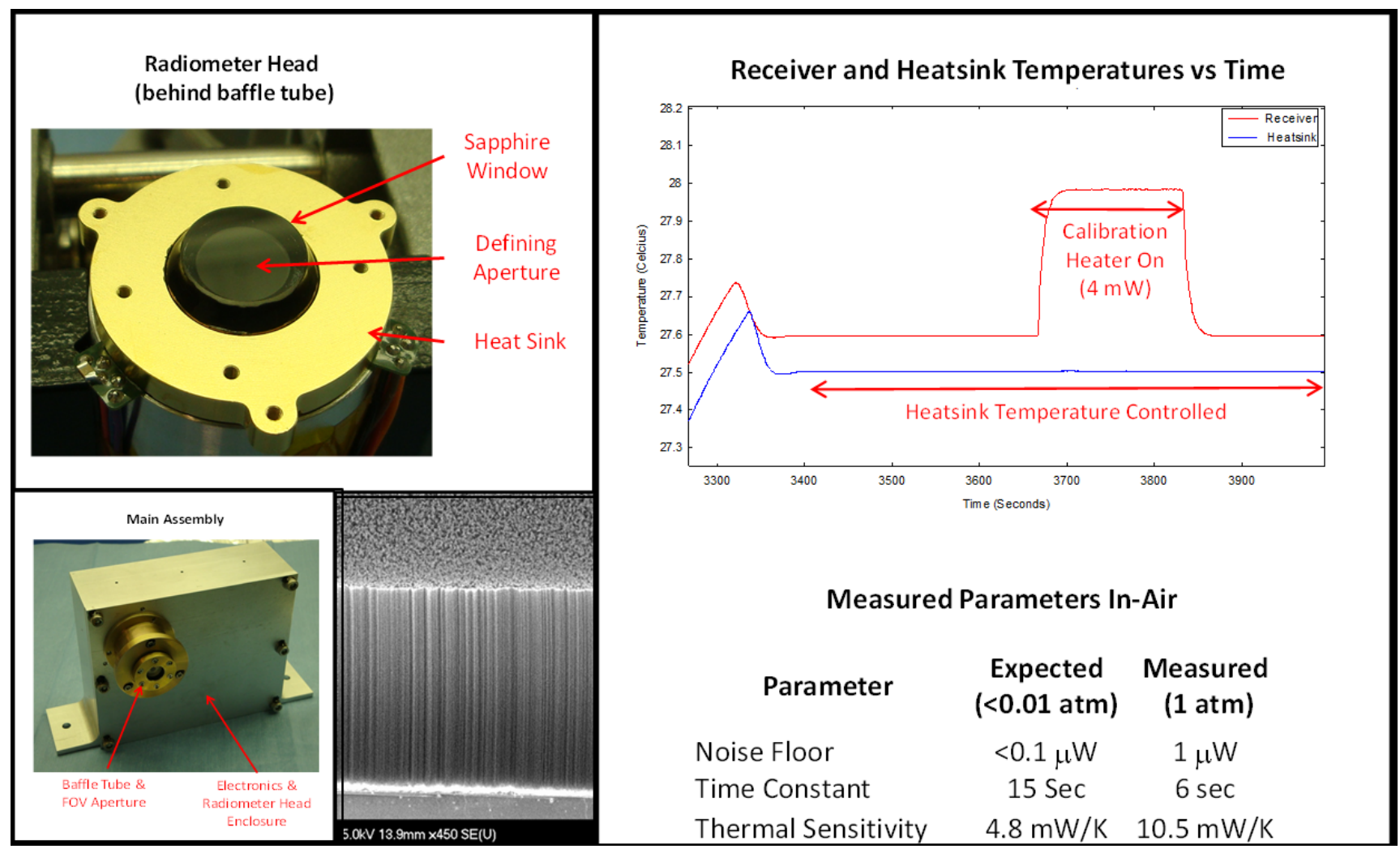

Figure 9. Top left: VACNT radiometer head. Bottom left: Radiometer payload assembly. Bottom center: Growth from rectangular patterned catalyst region of VACNT substrate. Right: Summary of radiometer test results.

\section{Technology Demonstration of Graphene Foils}

APL's second Phase 2 mission is funded by NSA FOP and involves a technology demonstration of graphene foils for integration into particle detectors for observations of planetary atmospheres, including Earth's. Graphene is a material with a number of unique qualities, including low permeability and high strength combined with low mass, but as a particle detector membrane, this technology currently resides at TRL 3. Through this proposed technology demonstration we intend to raise the TRL of graphene membranes to seven.

Many particle detectors use traditional carbon foils in the entrance aperture to help identify incoming particles, as well as to select the type of particles allowed to enter (Hamilton et al., 1990; Young et al., 1991). We are highly motivated to apply graphene membranes on particle detectors because they provide noticeably reduced foil mass and decreased power requirements over traditional carbon foils (Graf et al. 2007; Koenig et al. 2011, 2012). These advantages, when combined with anticipated performance improvements, could enable critical scientific measurements where mission cost, performance, or TRL limited such graphene application. For this experiment, we worked closely with Luxel Corporation to grow graphene test foils (funded for this project through the NASA SBIR program), and we conducted ground tests of their charged particle transmission factors as well as scanning electron microscope (SEM) imaging to examine microscopic structure (Figure 10). Remapping showed the graphene coverage to be $89 \%$ to $95 \%$ after transport, SEM cycles, and $\mathrm{Ar}^{+}$bombardment compared, with $88 \%$ to $96 \%$ as-made.

The foils are contained in individual enclosures (Figure 10) that will be integrated on JANUS, which will be mounted externally on the Blue Origin New Shepard PM interface for a flight test tentatively in late 2021. JANUS will provide environmental monitoring while the foils experience the full range of spaceflight stresses.

In preparation for this flight demonstration, we researched and developed a method of fabricating graphene membranes. The process produced grid-supported single-layer graphene (SLG) membranes on nanohole arrays (with hole diameters in $150-400 \mathrm{~nm}$ range) with hole coverage of $99 \%$. The nanohole arrays, with hole diameters in 150-400 nm range and an open fraction $\sim 8 \%$, were also highly efficient Ly-a $(122 \mathrm{~nm})$ blockers, with a demonstrated transmission of $\sim 0.2 \%$. We also developed a method for attaching SLG to $\mathrm{Ni}$ without 


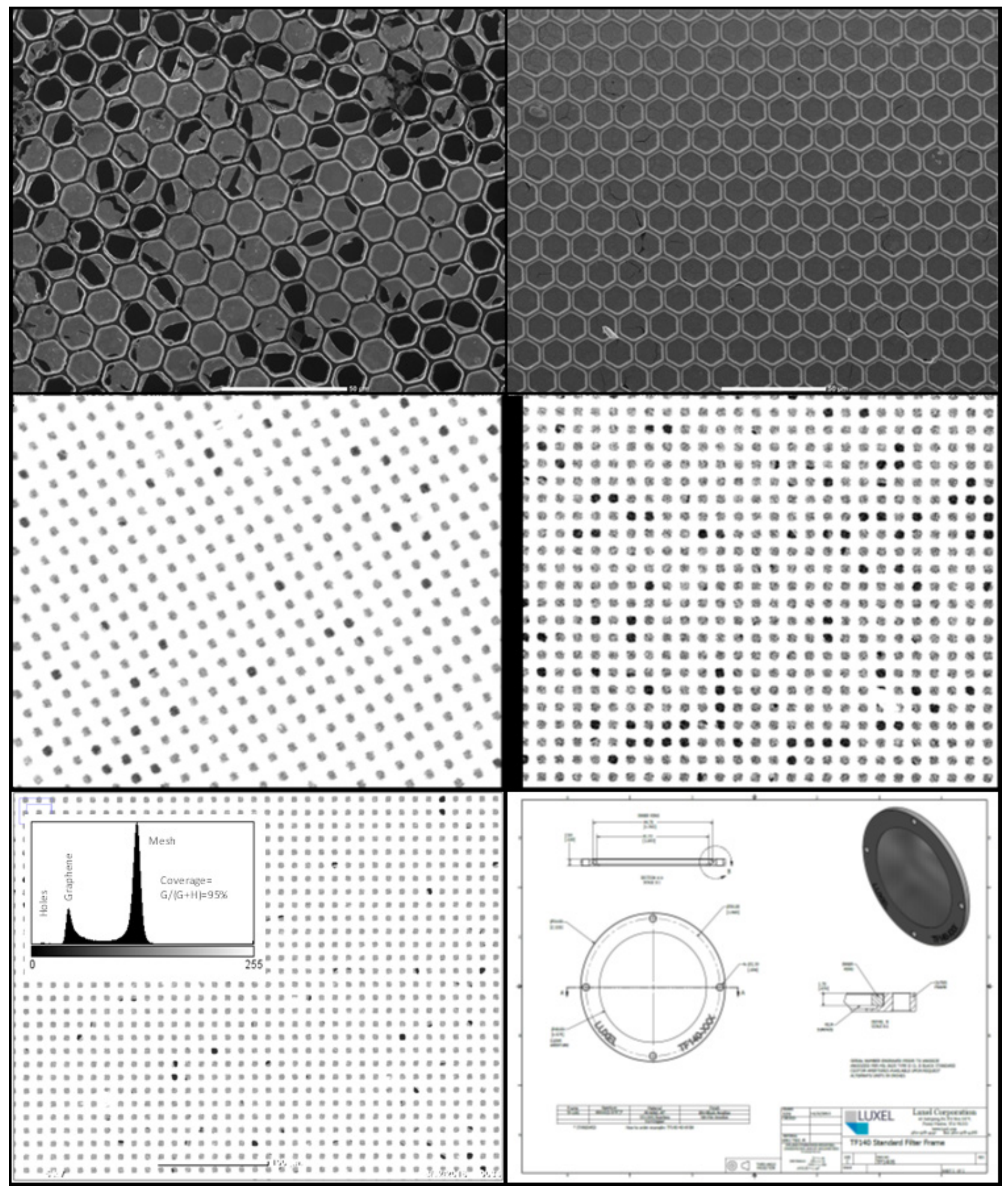

Figure 10. Top: Single-layer graphene attached to lithographically patterned Al 3 micron wide mesh (Left panel: A bad region with substantial cell breakage. Right panel: A good region with essentially all cells intact). Middle left: Single-layer graphene \#G13 on 2000 Ipi Ni mesh asmade. Middle right: Sample after 90 days, two cross-country FEDEX trips, 3 cycles into an SEM, and a few days of $10 \mathrm{keV} A r+$ ion bombardment. Bottom left: Detection of single-layer graphene \#G15 using SEM (inset shows gray levels associated with the mesh, the graphenecovered cells, and holes). Bottom right: Foil test flight enclosure. 


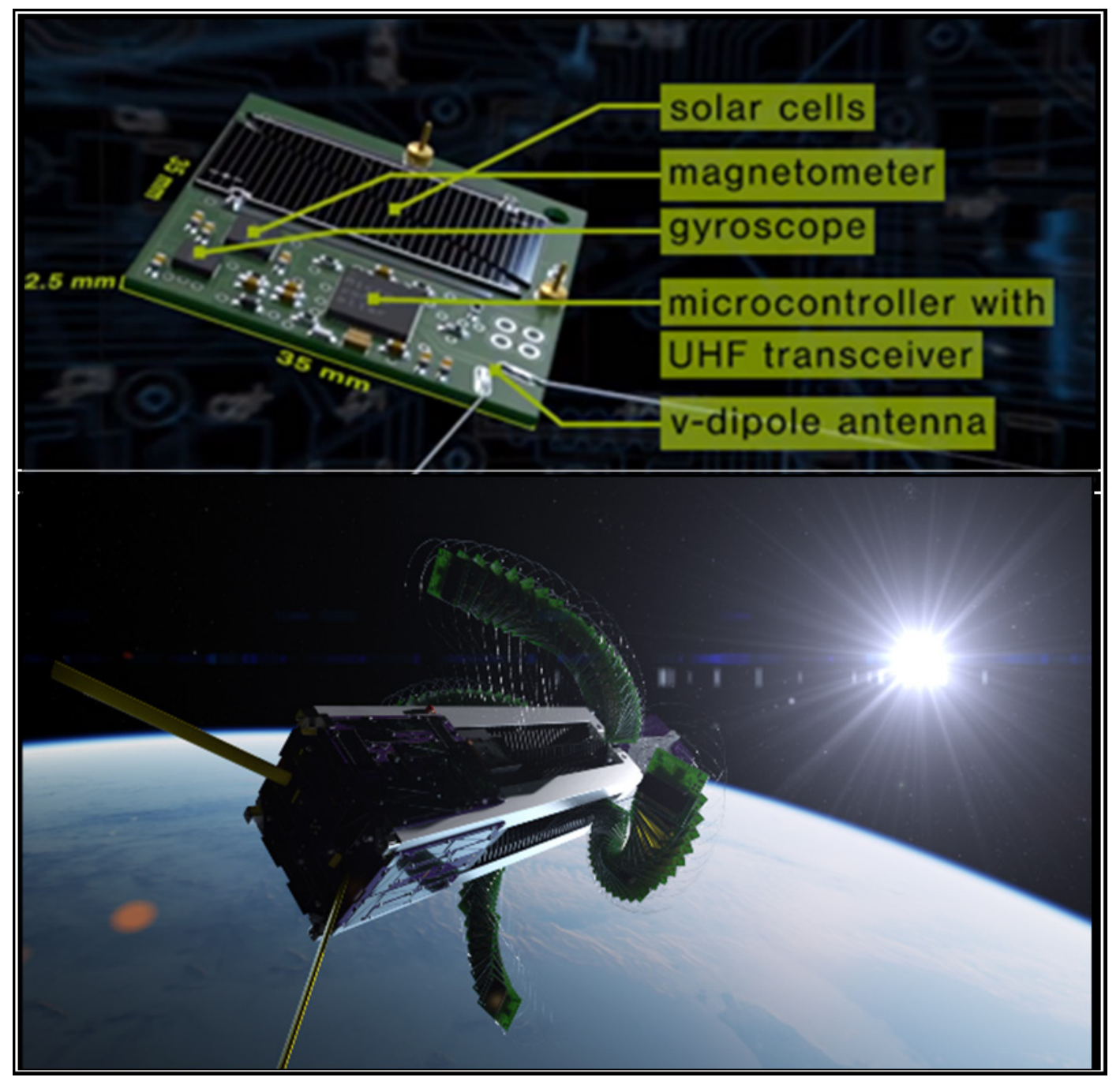

Figure 11. Top: Schematic of $200 \mathrm{~g}$ ChipSat. Bottom: TRL 7 ChipSat Deployer on a $1 U$ CubeSat to be used during the suborbital flight test (Image Courtesy of Ben Bishop).

adhesive and bilayer graphene (BLG) membranes with $>95 \%$ coverage on Ni mesh, achieving areas up to $10 \mathrm{~cm}^{2}$ on the 8 micron pitch and $40 \%$ open area hexagonal mesh (Zeiger et al., 2019)

Once these graphene foils were fabricated, we conducted a simple experiment to verify the integrity of the samples. In a vacuum system, we set up a particle beam experiment with an ion beam gun, pinhole collimators attached to a motion stage, and a Quantar beam imager. The ion beam composition is an admixture of protons, nitrogen, and other air-like constituents and can span an energy range from hundreds of eV to $10 \mathrm{keV}$. The pinhole collimator system consists of a rectangular aluminum plate with two pinholes approximately $\sim 1 \mathrm{~mm}$ in diameter at a distance of $88 \mathrm{~mm}$ upstream of the beam imager. Graphene samples to be tested are mounted over one pinhole, while the other is left open. The motion stage slews the collimator with and without the graphene sample in and out of the beam. The beam imager is made by Quantar and consists of a $70 \mathrm{~mm}^{2}$ open face microchannel plate (MCP). On the backside of the MCP is a position-encoding anode. During the experiment the vacuum pressure was $1^{\prime} 10^{-7}$ torr. The test verifies graphene coverage of the mesh support. Based on the results from test foils, we fabricated two sets of two flight test foils (four in total). One set will be exposed to atmosphere and the other will be held in vacuum during launch.

We intend to measure charged particle transmission through the exposed foils into individual Faraday Cups (FC) during flight. We will then retrieve these foils after flight and reproduce the previous ground tests to examine performance and possible damage caused by flight. This will help provide 
insight into the suitability of these foils for future particle detection instruments.

\section{Suborbital Flight Testing of Chip-Scale Satellites}

The next NASA FOP-funded JANUS-supported flight test is a technology demonstration of chip-scale satellite (ChipSat) deployment from a commercial reusable launch vehicle. More specially, our project will conduct a flight test of the JANUSChipSat payload on a Blue Origin New Shepard launch vehicle to raise TRL for future Earth and lunar/planetary missions. A ChipSat is a very small, open-source, 2-4 gram, free-flying TRL 7 (4 gram version) spacecraft with actuators, solar panels, sensors, processing, communications, and structure, as well as power suitable for a wide variety of small-scale payloads (Figure 11). Our goal is to validate operational capabilities as well as assess the reentry survival rate of ChipSats that will be deployed from the external payload location on top of the Blue Origin New Shepard Propulsion Module.

These "ChipSat" (sometimes referred to as femtosatellites on the order of less than $100 \mathrm{~g}$ ) can be produced in large quantities at very low cost, which offers the possibility of deploying hundreds or thousands of such satellites to provide coincident coverage on spatial scales previously unimagined (Streetman et al., 2015; Gilster, 2017; Howard, 2017). Development and testing of this new class of satellites has only recently emerged, and key open questions remain about their functionality as a network and their ability to return samples and data through the atmosphere without appreciable signal degradation. Thus, the TRL of these devices needs to be improved before they can be applied operationally for missions that exploit these capabilities.

A 2011-2014 experiment on the International Space Station (MISSE-8) validated the architecture and verified the performance of key components in LEO. Next, the KickSat-1 spacecraft (Manchester et al., 2013) was launched in 2014 with 104 ChipSats; however, the spacecraft did not successfully deploy them. More recently, members of our team participated in the successful KickSat-2 mission in March 2019, which achieved successful deployment of 105 four gram ChipSats.

For this mission, we will deploy 100 improved (2 gram) ChipSats with GPS and networking capability, not only to test operational measurement capabilities but also to conduct the first ever test of survival capabilities under suborbital reentry. We will then use satellite GPS signals to recover them from the landing site and analyze their survivability rate. We will deploy the ChipSats just after apogee of the suborbital flight, each capable of reporting attitude and position (GPS) data along with temperature, pressure, and magnetic-field measurements. They will be released through the flightheritage deployer design triggered by JANUS, which is roughly the size of a $3 U$ CubeSat (Figure 11), but they will not gain appreciable lateral acceleration, and they will descend along with the PM. JANUS will also record the usual environmental observations to combine with the ChipSat individual data. The ChipSats' low ballistic coefficient may disperse them up to several miles from the launch site, yielding valuable, firstever data of this type on upper-atmospheric winds and other environmental phenomena. The ChipSats continually transmit their GPS-derived position, so they can be located and collected after they land. The results from this flight test will provide critical information on the survivability and operation of these ChipSats for suborbital applications and help raise TRL for orbital (and beyond) applications.

\section{Suborbital Flight Testing of Charged Particle Technology} NASA FOP has also selected an APL mission to conduct a suborbital flight test of a charged particle instrument being developed for the Europa Clipper instrument. The test also facilitates establishment of the first external in situ particle observations on the Blue Origin New Shepard SRLV. A goal of this mission is to develop a low cost, reliable method of making in situ particle observations at suborbital between 60 and $120 \mathrm{~km}$. This region, while extremely important for understanding our atmosphere, still contains many mysteries because it has been difficult to access. It is not easily accessible due to logistical constraints, i.e., too high for conventional aircraft and balloons, too low for orbiting satellites. However, it is an important region of Earth's atmosphere encapsulating two important atmospheric boundaries: the "mesopause" and "turbopause"-these encompass the altitudes where atmospheric temperature is lowest and the chemical constituents cease to be well mixed-both critical in determining the Earth's temperature and chemical profile. Ion chemistry at the Earth is complex and variable but we have made limited progress in quantifying its variability, either spatially or temporally (Arnold et al., 1971) at the proposed suborbital altitudes.

This flight test of our payload will enable a better understanding of this critical transition region of our atmosphere. This involves application of a suborbital variation of the Plasma Instrument for Magnetic Sounding (PIMS) shown in Figure 12. PIMS, scheduled to be flown on the NASA Europa Clipper mission, is primarily a FC with a $90^{\circ}$ FOV measuring the 1.5-dimensional velocity distribution function of ions and electrons. This FC measures the current produced on metal collector plates by charged particles with sufficient energy per charge (E/q) to pass through a modulated retarding grid placed at variable (AC) high voltage $(\mathrm{HV})$. Figure 12 also shows the characteristics of this sensor (note for the proposed flight test, we only require a test of the low energy, or "ionospheric," mode).

The key focus of this flight test will be integration with the JANUS system on the external mounting location on the New 


\begin{tabular}{|l|l|}
\hline \\
\hline Magnetospheric Mode \\
Energy Range
\end{tabular} \begin{tabular}{l}
$0.05-2.0 \mathrm{keV}$ (electrons) \\
\hline lonospheric Mode \\
Energy Range
\end{tabular}

Figure 12. Top left: PIMS Faraday Cup sensor. Top right: PIMS electronics assembly/bank. Bottom: PIMS operational constraints.

Shepard PM. We will conduct measurements of electrons and negative ions during all phases of the flight. The intent will be to enable these observation during all subsequent flights of JANUS. Such observations will be the first analysis of charged particles in a specific suborbital region over many different time periods affording us unprecedented temporal and spatial insight in this atmospheric region.

\section{SUMMARY}

While originally targeting space tourism, commercial reusable suborbital launch vehicles offer game-changing capabilities for scientific research as well as instrument development and technology demonstration. Key aspects of these revolutionary spacecraft include dramatic reduction in cost over existing suborbital platforms and a guaranteed safe return of payload on launch day. The JHU APL has established a CSP to rapidly exploit these new vehicles. The core of this program is the JANUS system that provides common payload capabilities for power delivery, data logging, event triggering, and environmental monitoring, eliminating the need to develop an entire new payload for each flight. JANUS has already flown six successively more complex flight tests of this system on Masten Space Systems, Blue Origin, and Virgin Galactic vehicles (funded by NASA and APL) to develop the JANUS system and characterize vehicle environments. All data is available upon request. APL is now preparing to conduct five operational technology demonstration and research missions. These activities will not only develop novel applications for these new vehicles but also allow for support of much larger missions on commercial orbital launch systems as well as commercial lunar landers.

\section{ACKNOWLEDGMENTS}

This work is supported by the NASA Science Technology Mission Development Flight Opportunities Program and Game Changing Opportunities in Technology Development Program as well as the Johns Hopkins Applied Physics Laboratory Internal Research and Development Program. The graphene development was funded at Luxel Corporation through NASA SBIR NNX17CG14C.

\section{REFERENCES}

Arnold F, Kissel J, Krankowsky D, Wieder H, Zähringer J (1971) Negative ions in the lower ionosphere: a mass-spectrometric measurement. Journal of Atmospheric and Solar-Terrestrial Physics 33(8), 1169-1170.

Arnold SP, Smith HT (2013) Suborbital flights for space science. In AIAA SPACE 2013 conference and exposition, September 10-12, 2013, San Diego, CA, doi: 10.2514/6.2013-5350. 
Barucci MA, Cheng A, Michel P, Benner L, Binzel R, Bland P, Böhnhardt H, Brucato J, Bagatin AC, Cerroni P, et al. (2011) Marcopolo near earth asteroid sample return mission. Experimental Astronomy 33(2-3), 645-684.

Ceplecha Z, Borovička J, Elford W, Revelle D, Hawkes R, Porubcan V, Simek M (1998) Meteor phenomena and bodies. Space Science Reviews, 84, 327-471.

Christe S, Zeiger B, Pfaff R, Garcia M (2016) Introduction to the special issue on sounding rockets and instrumentation. Journal of Astronomical Instrumentation 5(1), id. 1602001-503, doi: 10.1142/ S2251171716020013.

Cola B, Xu X, Fisher T (2007) Increased real contact in thermal interfaces: a carbon nanotube/foil material. Applied Physics Letters 90, 093513.

Cooper C, Mitchell CN, Wright CJ, Jackson DR, Witvliet BA (2019) Measurement of ionospheric total electron content using singlefrequency geostationary satellite observations. Radio Science 54, 10-19. doi: 10.1029/2018RS006575.

Cziczo D, Thomson D, Murphy D (2001) Ablation, flux, and atmospheric implications of meteor inferred from stratospheric aerosol. Science 291, 1772-1775.

Dyrud L, Woessner B, Jovancevic A, Ganguly S (2007) Ultra tightly coupled GPS/INS receiver for TSPI applications. In Proceedings of ION GNSS 2007, Fort Worth, TX.

Gilster P (2017) Breakthrough Starshot 'Sprites' in Orbit," Centauri Dreams, July 27, 2017.

Graf D, Molitor F, Ensslin K, Stampfer C, Jungen A, Hierold C, Wirtz L (2007) Spatially resolved Raman spectroscopy of single- and few-layer graphene. Nano Letters 7(2), 238-242.

Hamilton DC, Gloeckler G, Ipavich FM, Lundgren RA, Sheldon RB (1990) New high-resolution electrostatic ion mass analyzer using time of flight. Review of Scientific Instruments 61, 3104.

Howard CE (2017) "Winning femtosatellite launch device design 3D-printed in space, now in orbit." Intelligent Aerospace; April 20, 2017. Retrieved from: https://www.intelligent-aerospace.com/ articles/2017/04/winning-femtosatellite-launch-device-design-3dprinted-in-space-now-in-orbit.html (Accessed July 28, 2018).

Hunten DM, Turco RP, Toon OB (1980) Smoke and dust particles of meteoric origin in the mesosphere and stratosphere. Journal of the Atmospheric Science 37, 1342-1357, doi: 10.1175/1520-0469(1980)037<1342:SADPOM>2.0.CO;2.

Jurist JM (2009) Commercial suborbital sounding rocket market: a role for reusable launch vehicles. Astropolitics 7(1), 32-49, doi: 10.1080/14777620902782660.

Koenig SP, Boddeti NG, Dunn ML, Bunch JS (2011) Ultrastrong adhesion of graphene membranes. Nature Nanotechnology 6(9), 543-546.

Koenig SP, Wang L, Pellegrino J, Bunch JS (2012) Selective molecular sieving through porous graphene. 1-5.

Manchester Z, Peck M, Filo A (2013) KickSat: a crowd-funded mission to demonstrate the world's smallest spacecraft. In AIAA/ Utah State University Small Satellite Conference, Logan, UT.
Mizuno K, Ishiib J, Kishidac H, Hayamizua Y, Yasudaa S, Futabaa DN, Yumuraa M, Hataa K (2009) A black body absorber from vertically aligned single-walled carbon nanotubes. Proceedings of the National Academy of Sciences of the United States of America 106(15), 6044-6047.

Rymer AM, Smith HT, Wellbrock A, Coates AJ, Young DT (2009) Discrete classification and electron energy spectra of Titan's varied magnetospheric environment. Geophysical Research Letters 36(15), doi: 10.1029/2009GL039427.

Sample JL, Rebello KJ, Saffarian H, Osiander R (2004) The ninth intersociety conference on thermal and thermomechanical phenomena in electronic systems. 297-301.

Sauser B, Ramirez-Marquez J, Dinesh V, Gove R (2006) From TRL to SRL: the concept of systems readiness levels. In Conference on Systems Engineering Research, Los Angeles, CA, April 7-8, 2006, Paper \#126.

Smith HT, Rymer AM (2014) An empirical model for the plasma environment along Titan's orbit based on Cassini plasma observations. Journal of Geophysical Research 119, 2014JA019872.

Streetman B, Shoer J, Peck M (2015) Exploration architecture with quantum inertial gravimetry and in situ ChipSat sensors. NIAC Phase 1 Final Report, 2015.

Sukkarieh S, Nebot EM, Durrant-Whyte HF (1999)A high integrity IMU/ GPS navigation loop for autonomous land vehicle applications. IEEE Transactions on Robotics and Automation 15(3)/.

Swartz WH, Lorentz SR, Papadakis SJ, Huang PM, Smith AW, Deglau DM, Yu Y, Reilly SM, Reilly NM, Anderson DE (2019) RAVAN: CubeSat demonstration for multi-point earth radiation budget measurements. Remote Sensing 11(7), 796. doi: 10.3390/ rs11070796.

Xu J, Fisher TS (2006) Enhanced thermal contact conductance using carbon nanotube array interfaces. IEEE Transactions on Components and Packaging Technologies 29(2), 261-267.

Yang Z-P, Lijie C, Bur JA, Lin S-Y, Ajayan PM (2008) A vertically aligned carbon nanotube array: the darkest manmade material. Nano Letters 8, 446.

Young DT, Barraclough BL, McComas DJ, Thomsen MF, McAbe K, Vigil R (1991) CRRES low-energy magnetospheric ion composition sensor. Journal of Spacecraft and Rockets 29, 596-598.

Zeiger BR, Shields MW, Paul B, Lopez H, Grove D, Arrowsmith W, Smith R, Primus C, Ayers T (2019) Graphene foils for neutral atom detectors. In Proceedings of SPIE 11127, Earth Observing Systems XXIV, 111272C (9 September 2019), doi: 10.1117/12.2529762. 